\title{
Survey of Hearing Aid Conditions for Hearing Impaired Persons While Driving
}

\author{
Hiroshi Ikeda* \\ Advanced Research Institute for Science and Technology, Osaka City University, Japan \\ Email: h-ikeda@osa.att.ne.jp \\ Shigeyuki Minami \\ Advanced Research Institute for Science and Technology, Osaka City University, Japan
}

\author{
Article History \\ Received: September 15, 2020 \\ Revised: October 25, 2020 \\ Accepted: October 27,2020 \\ Published: October 30, 2020
}

\begin{abstract}
Hearing impaired persons are required to drive with hearing aids to supplement their hearing ability, however, there has not been sufficient discussion regarding the impact of the use of a hearing aid on driving a vehicle. In order to investigate the actual usage and driving conditions of using hearing aids while driving a vehicle, this paper uses a questionnaire to survey (1) how easy it is to drive when wearing hearing aids, and (2) how often hearing aids are not worn while driving. Concerning the ease of driving when wearing a hearing aid, it was suggested that people with congenital hearing loss were more likely to rely on visual information, and those with acquired hearing loss continue to use their experience of hearing. When the level of disability is high, it is difficult to drive when using the hearing aid, and when the disability level is low, it is easier to drive. Regarding the frequency of driving without wearing hearing aids, about $60 \%$ of respondents had such an experience. Those who often drive without hearing aids had experienced headaches due to noise from wearing hearing aids compared to those who wear hearing aids at all times. Hearing aids are necessary assistive devices for hearing impaired persons to obtain hearing information, and to provide a safe driving environment. Therefore, this paper addresses issues to maintain a comfortable driving environment while wearing a hearing aid.
\end{abstract}

Keywords: Hearing impaired; Hearing aid; Vehicle; Driving; Noise.

\section{Introduction}

In 1919, Japan's first car driving laws came into force. Among them, "psychotic, deaf, and blind" people were disqualified from issuance of a driver's license. Although this law was abolished in 1947, the "Road Traffic Law" was enacted in 1960, and Article 88 states that "psychotic, mentally retarded, epileptic, blind, and deaf, or those who do not speak or who cannot speak are targeted for refusal to obtain a license". However, a national movement began in the 1950s in search of the right to drive a vehicle freely, and since 1973, it has been approved to wear hearing aids in the aptitude test of the ordinary license test. A driver's license will be issued to a person with hearing impairment under the condition that "you can hear a sound of 90 decibels 10 meters away with a hearing aid attached".

However, with regard to the conditions for obtaining a license, many hearing impaired persons are demanding a review of their hearing aid obligations and aptitude tests. The reason for this is that it is possible to drive without hearing information if there is visual information, and there are many countries where hearing impairment is not subject to license restrictions. In addition, some hearing impaired persons want to test their ability to collect information visually rather than through hearing information. Due to such circumstances, from June 2008, even without wearing hearing aids, it is permitted for hearing impaired persons to drive ordinary passenger cars under the condition that they display a special mark for hearing impairment, as shown in Figure 1, and a specific rear-view mirror is attached.

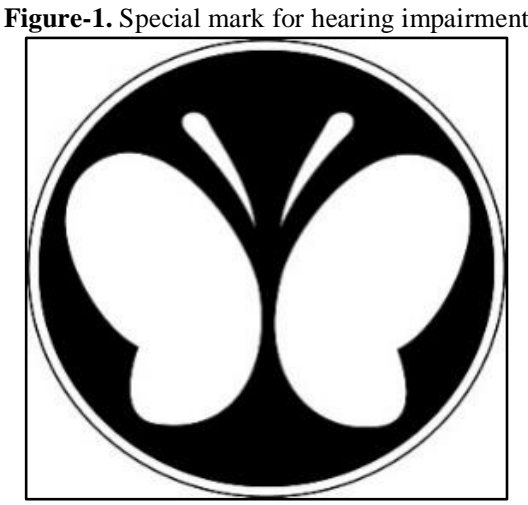




\section{Sumerianz Journal of Social Science}

Hearing impaired persons are required to drive with a hearing aid to supplement their hearing, and there are various types of hearing aids such as an analog type, digital type, and program type. Hearing aids have a long history, and initially, people listened through animal horns and shells. Later, something called an ear trumpet was used by inserting it into the ear. There are various sizes and shapes, and this type was also used by Beethoven in his later years. The ear trumpet continued to be researched and devised, and the first industrialized version was by Frederik C. Rein, which is said to have been factory-produced in London from around 1800 (Berger, 1970).

A modern type of hearing aid was created around 1900 (Ikeda, 2013). Since this was originally an application of telephone technology, it can be said that the beginning of electrical hearing aids was in 1876, when Alexander Graham Bell invented the telephone. Later, the transistor invented by William B. Shockley et al. in 1948 brought a breakthrough in hearing aids (Mills, 2011). With the miniaturization and generalization of hearing aids, it has become possible to drive a vehicle even with hearing impairment if a hearing aid is used.

However, there is not enough discussion about the influence of hearing aids on hearing impaired persons while driving a vehicle. Excessive noise is the most common reason for not wearing hearing aids when driving (Kochkin, 2002). In addition, according to a survey by Nakagawa and Nagahara (1999) examining the degree of satisfaction with hearing aids under noise, people in Japan are less satisfied than people in the U.S.A. They explained that the reason for this is that hearing aid fitting technology is lower in Japan.

In the current situation where hearing aids are not satisfying for the hearing impaired when driving a vehicle, it is more important to create an environment where people with disabilities can drive safely rather than to exclude people with disabilities from using a vehicle. It is necessary to investigate the actual usage of automobiles, because a car is more convenient as a means of transportation for the hearing impaired than public transportation. Therefore, the purpose of this study is to investigate the actual usage and driving situation of the hearing impaired using hearing aids while driving a vehicle, in order to understand the current situation and problems from the viewpoint of consciousness, and to clarify future issues.

\section{Method}

A questionnaire was used to investigate the actual usage and driving situation of hearing impaired persons using hearing aids while driving a vehicle. Specifically, in addition to individual attributes, two questions were conducted regarding (1) the driving sensation when using a hearing aid, and (2) the frequency of wearing a hearing aid while driving. The subjects and procedures of the survey are as follows:

\subsection{Subjects}

It is difficult to classify and define hearing impaired persons because of the various causes and types of hearing impairment and the degree of hearing. In this study, hearing impaired persons are classified into "congenital hearing loss", which means that they have impairment from birth, and "acquired hearing loss", which means that they became impaired after they were born. In addition, there are many types of hearing aids, and their performances are also different, so they were excluded from the question items.

The total number of valid responses obtained from this questionnaire survey was 91 . Based on the survey results by Nihon Hochouki Kougyoukai (2018) that $80 \%$ of hearing aid owners have hearing loss in both ears, this questionnaire targeted people with a driver's license and hearing loss in both ears. The average age of the respondents was 49.5 years $(\mathrm{SD}=12.5)$, with $67.0 \%$ men and $33.0 \%$ women. The average number of years since obtaining a driver's license was 23.1 years $(\mathrm{SD}=9.2)$. Regarding the content of disability, $53.8 \%$ had congenital hearing loss and $46.2 \%$ had acquired hearing loss. The disability level was $25.3 \%$ for Level $1,54.9 \%$ for Level 2, and $19.8 \%$ for Level 3 .

\subsection{Investigation Procedure}

The questionnaire was distributed to the subjects under the supervision of organizations related to the hearing impaired in $\mathrm{O}$ City and $\mathrm{K}$ City, and after completion, they were mailed to a designated address. The question items were created after a prior meeting to understand the driving environment of the hearing impaired. It should be noted that the answers to the questions are a selection method, and if there was no intention to answer, it was not necessary. Also, in order to protect the privacy of the respondents, the envelope containing the questionnaire was submitted after being sealed by themselves.

\section{Results}

When asked about how easy it is to drive with a hearing aid, the answer was chosen from three choices: "easy to drive", "no change", and "difficult to drive". $37.5 \%$ of the respondents answered that it was "easy to drive" with hearing aids, and $26.1 \%$ answered that it was "difficult to drive". However, the respondents who said "no change" showed the highest value at $36.4 \%$.

Therefore, when comparing the ease of driving with the use of hearing aids under the conditions of congenital hearing loss and acquired hearing loss, as shown in Figure 2, those who answered that it was "easy to drive" was $25.5 \%$ for congenital hearing loss and a higher response rate of $51.2 \%$ for acquired hearing loss. On the other hand, those who answered that it was "difficult to drive" was $34.0 \%$ for congenital hearing loss and $17.1 \%$ for acquired hearing loss, the former showing higher value. The results indicate that the ease of driving with hearing aids can be said to be related to the time of disability $\left(\chi^{2}=4.46, d f=1, p<0.05\right)$.

Regarding the ease of driving with hearing aids by level of disability the respondents in Level 1, with the heaviest disability, $43.5 \%$ answered that driving was "difficult". On the other hand, in Level 3, where the level was 
light, $55.6 \%$ respondents answered that driving was "easy", however, there was no statistically significant difference.

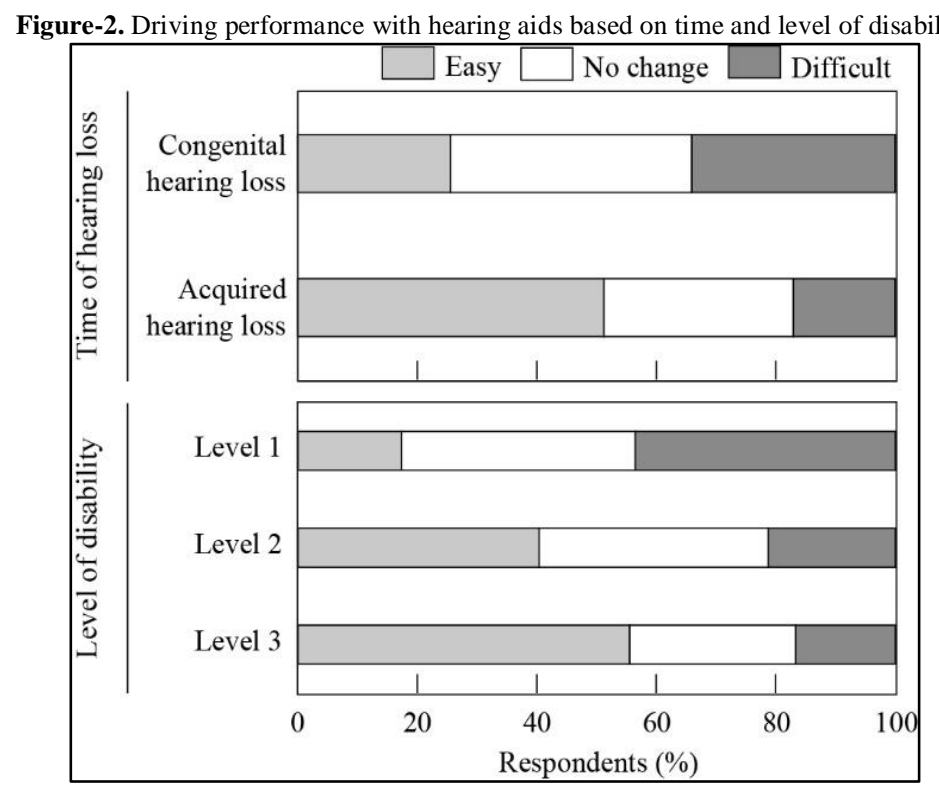

Next, the respondents were asked about the frequency of not wearing hearing aids while driving. Answers were chosen from three: disuse is "frequent", "sometimes" and "not at all". As a result, $41.6 \%$ of the respondents answered that it is "frequent" for them to drive without wearing hearing aids, $23.6 \%$ answered "sometimes" and $34.8 \%$ answered that disuse is "not at all".

When comparing the frequency of not using hearing aids between congenital hearing loss and acquired hearing loss, as shown in Figure 3, among those who answered that not using hearing aids was "frequent", the congenital hearing loss had a higher response rate of $49.0 \%$, and the acquired hearing loss had a rate of $32.5 \%$. On the other hand, for those who answered that the frequency of not using a hearing aid was "not at all", congenital hearing loss was $24.5 \%$ and acquired hearing loss was $47.5 \%$, the latter showing higher values. Regarding the frequency of not using hearing aids by level of disability, $60.9 \%$ of the respondents answered that the disuse was "frequent" at Level 1 with the heaviest disability. On the other hand, in Level 3, where the level was light, the largest number of respondents answered that disuse was "not at all", at $44.4 \%$. However, there was no statistically significant difference in both the time of disability and the level of disability.

Figure-3. Hearing aid disuse frequency from time and level of disability

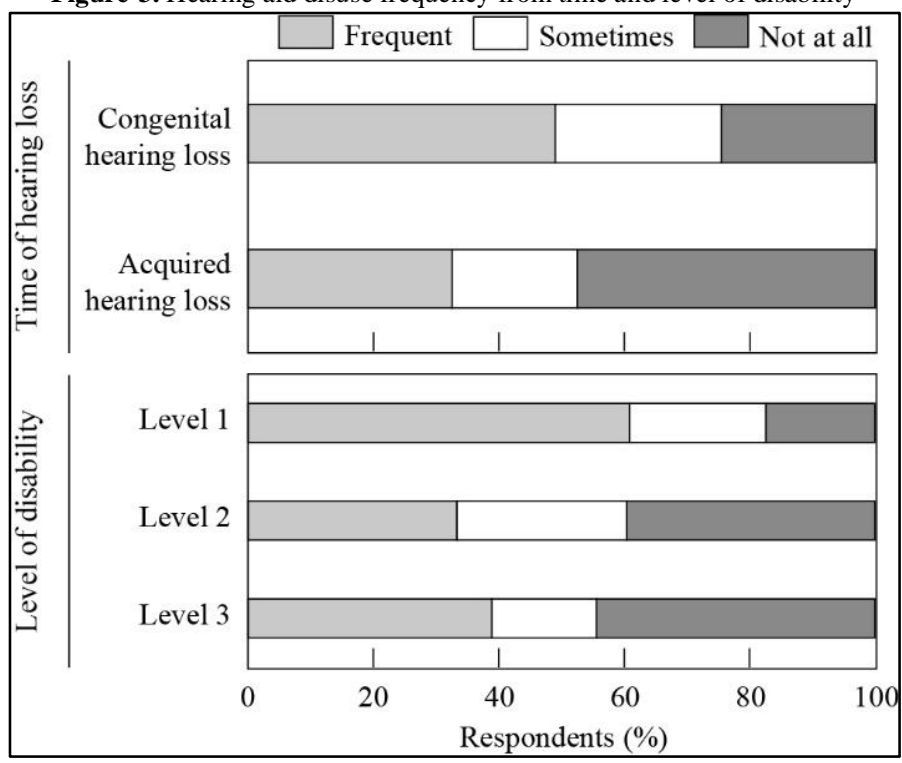

Figure 4 compares the ease of driving with the use of a hearing aid and the frequency of wearing a hearing aid during driving. Of those who answered that it was "easy to drive", the hearing aid disuse was low in frequency, with $11.1 \%$ having a "frequent" disuse of a hearing aid, $33.3 \%$ having a disuse "sometimes", and 55.6 \% with disuse "not at all". On the other hand, those who answered that it was "difficult to drive" were $67.7 \%$ of those with "frequent" disuse, $22.6 \%$ with "sometimes" disuse and $9.7 \%$ with disuse "not at all". It can be said that the ease of driving with the use of hearing aids is related to the frequency of wearing hearing aids $\left(\chi^{2}=29.67, d f=2, p<0.01\right)$. 
Sumerianz Journal of Social Science

Figure-4. Ease of driving when using hearing aids and frequency of disuse

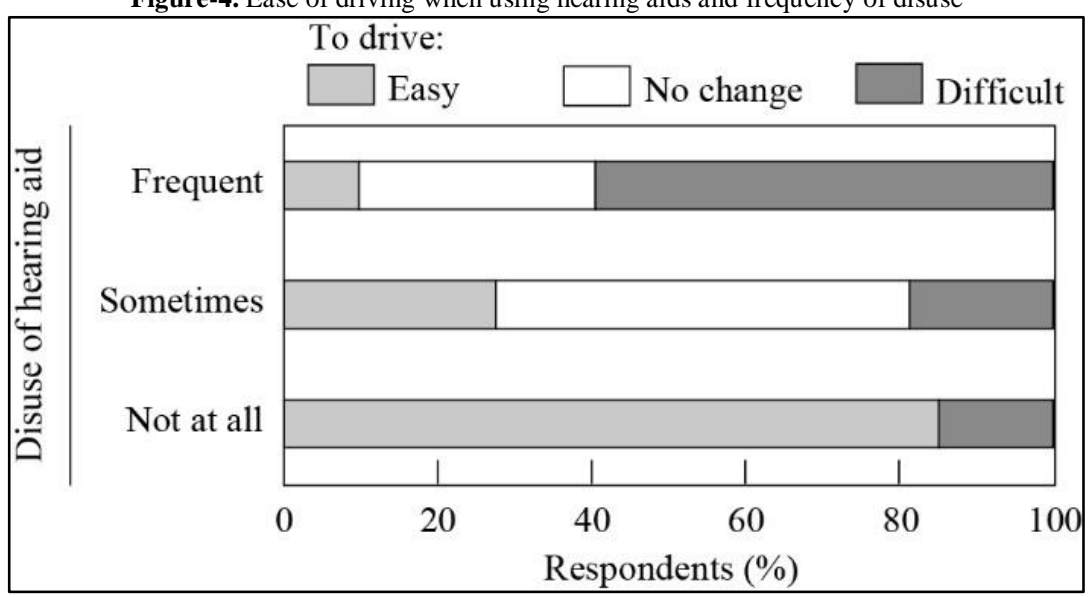

One possible reason for not using hearing aids is that noise, such as engine noise, may make the user feel uncomfortable. According to a survey conducted by the Japanese Federation of the Deaf (Japanese Federation of the Deaf, 2006), only one third of all hearing impaired people use hearing aids when driving a vehicle. Therefore, the respondents were asked about the relationship between removing the hearing aid or adjusting the volume when feeling uncomfortable due to noise. As a result, $22.6 \%$ of the respondents answered that disuse is "frequent", $21.4 \%$ answered "sometimes", and $56.0 \%$ answered "not at all".

Therefore, when compared with the frequency of disuse of hearing aids, a statistically significant difference was found $\left(\chi^{2}=23.60, d f=2, p<0.01\right)$. As shown in Figure 5, those who showed the largest proportion were those who answered that it is "frequent" to remove the hearing aid or adjust the volume due to feeling uncomfortable with noise etc. while driving, $84.2 \%$ of the respondents said that they frequently do not use hearing aids. In addition, the next largest proportion, $55.3 \%$, was those who answered "not at all" to remove the hearing aid or adjust the volume due to noise etc. while driving. It can be said that the use or removal of a hearing aid or adjusting the volume is related to feeling uncomfortable due to noise while driving.

Figure-5. Experience of hearing aid removal due to noise and frequency of hearing aid disuse

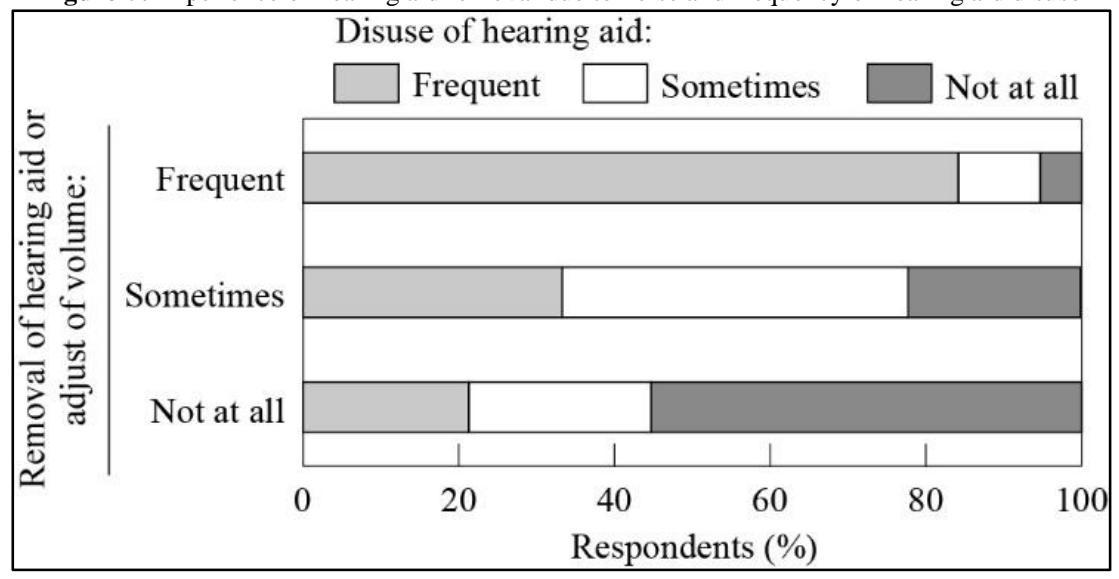

\section{Discussion}

As for driving when wearing a hearing aid, it was found that the ease of driving could be improved by about 30 $\%$. There was an opinion that the reason was that various sounds (horns, sirens, etc.) could be heard and the surrounding environment could be grasped. On the other hand, there was an opinion that the reason why the ease of driving is reduced is that noise, such as engine noise, makes the user feel uncomfortable and that unnecessary sounds such as exhaust noise of a bus or truck and rain can be heard. It was suggested that persons with congenital hearing loss were more likely to rely on visual information and that acquired hearing loss persons may continue to use their experience of hearing. Further, it is suggested that it is difficult to drive when using the hearing aid when the disability level is high, and conversely, for persons with a low level disability, it is easy to drive when a hearing aid is used.

When asked about the frequency of driving without wearing hearing aids, about $60 \%$ of the respondents said that they had experience of driving without hearing aids. One of the reasons is that noise from using hearing aids causes discomfort and headaches. If important information is missed by removing the hearing aid, it will affect the driving operation. A study by Iwasaki et al. (2003), which evaluated the audibility of analog and digital hearing aids, reported that speech intelligibility through digital hearing aids improves even in the presence of noise. Since the digital hearing aid can easily suppress noise, similar effects should be obtained when driving a vehicle. However, there were also opinions that: it was not due to a problem with the hearing aid; that hearing was not required for driving; and that driving was possible if there was vision. There is also the reason that hearing impaired persons do 
not use hearing aids because they acquire information necessary for driving by reading the surrounding environment, such as movements of other vehicles.

\section{Conclusion}

People who often drive without using hearing aids experience headaches due to excessive noise from hearing aids compared to those who wear hearing aids at all times. There is a research result by Federal Highway Administration (1998) that even non-hearing impaired persons have a high accident rate when the noise level is high. Therefore, by wearing a hearing aid, noise may be related to the issue of driving safety. Hearing aids are necessary auxiliary equipment for hearing impaired persons to obtain hearing information, and it is important to provide a safe driving environment in which a hearing aid can be comfortably worn. To that end, it is necessary to consider the following issues which were obtained in this survey.

- Since the importance of auditory information when driving varies depending on the time of hearing impairment, it is necessary for hearing impaired persons to educate themselves to understand the importance of auditory information. For that purpose, it is necessary to establish an educational approach and to collaborate with a license center.

- It is necessary to investigate the specific effects of noise while driving to see what problems current hearing aids have while driving. It is also important to investigate what events lead to noise.

As a future issue, it is necessary to conduct research to clarify the situation that affects driving, using an objective evaluation method, especially regarding the specific effects of noise during driving.

\section{References}

Berger, K. W. (1970). The hearing aid: Its operation and development. The national hearing aid society detroit. 2223.

Federal Highway Administration (1998). Role of driver hearing in commercial motor vehicle operation: An evaluation of the FHWA hearing requirement.

Ikeda, H. (2013). Support technology for transportation environment of handicapped people. Union Press: Osaka.

Iwasaki, S., Nagai, N., Nagura, M., Arai, M., Takeyama, M. and Mineta, H. (2003). Zatsuonfuka onbakensa niyoru dejitaruhochouki no hyouka [Evaluation of digital hearing aids by noise-loaded sound field inspection]. Audiology Japan, 46(2003): 471-72.

Japanese Federation of the Deaf (2006). Questionnaire report of deaf people and driving license, 2006. in Japanese.

Kochkin, S. (2002). MarkeTrakV: Why my hearing aids are in the drawer: The consumer's perspective. The Hearing Journal, 53(2002): 34-42.

Mills, M. (2011). Hearing aids and the history of electronics miniaturization. IEEE Annals of the History of Computing, 33(2011): 24-44.

Nakagawa, T. and Nagahara, T. (1999). Chokakusyogaisya no hochouki no jikohyouka, Self-evaluation of hearing aids for the hearing impaired. Audiology Japan, 42(1999): 471-72.

Nihon Hochouki Kougyoukai (2018). Research report of japantrak 2018. In Japanese. 